\title{
Eternal: Fault Tolerance and Live Upgrades for Distributed Object Systems *
}

\author{
L. E. Moser, P. M. Melliar-Smith, \\ P. Narasimhan, L. A. Tewksbury, V. Kalogeraki \\ Department of Electrical and Computer Engineering \\ University of California, Santa Barbara, CA 93106
}

\begin{abstract}
The Eternal system supports distributed object applications that must operate continuously, without interruption of service, despite faults and despite upgrades to the hardware and the software. Based on the CORBA distributed object computing standard, the Eternal system replicates objects, invisibly and consistently, so that if one replica of an object fails, or is being upgraded, another replica is still available to provide continuous service. Through the use of interceptors, Eternal renders the object replication transparent to the application and also to the CORBA ORB. Consequently, Eternal is able to provide fault tolerance, and live hardware and software upgrades, for existing unmodified CORBA application programs, using unmodified commercial-off-the-shelf ORBs.
\end{abstract}

\section{Introduction}

Many computer systems used in defense applications must operate continuously without interruption of service. Such systems must provide continuous service in the presence of hardware and software faults, hardware that has been repaired and returned to service, hardware that has been upgraded, and software that has been corrected or upgraded to provide improved service. A typical requirement for continuous service is that the computer system must generate its response within a prescribed deadline, perhaps five seconds, and the deadline must be respected even in the presence of a fault or an upgrade. Such a deadline requirement implies almost immediate recovery from a fault. The interval between events, in which the system fails to achieve such immediate recovery, is required to be many years. This demanding requirement is no longer unique to

* This research has been sponsored by the Defense Advanced Research Projects Agency in conjunction with the Air Force Research Laboratory Rome under Contract F3602-97-1-0248. defense systems; many e-commerce and e-business applications now have essentially the same requirement.

It is certain that every hardware unit will fail eventually. Many software components will also fail; indeed, in complex systems, software faults are substantially more frequent than hardware faults. Under conditions of warfare, computer system failures occur even more frequently because of damage, hostile intrusions, or use of hardware or software rushed into service without adequate testing. It is also likely that, within the lifetime of a major defense system, every hardware component will be replaced by different hardware, possibly completely different, and every software component will be replaced by enhanced software, also potentially very different. Under conditions of warfare, whatever hardware and software are available are what must be used; in a future information war, it may be necessary to modify the software very frequently to counter new strategies or attacks of the enemy, or to exploit opportunities or vulnerabilities.

Unfortunately, the current state-of-the-practice is inadequate in the reliability and recovery time that is achieved, in the ability to use heterogeneous hardware and software, in the ability to upgrade hardware or software without interruption of service, in the cost and difficulty of developing new software, and most particularly in the development timescale for new software. Fault-tolerant defense systems (and many are not) may require several minutes to recover, long enough for an enemy to detect the fault and to launch an attack to exploit it. Very few defense systems can exploit heterogeneous hardware and software. Even fewer defense systems attempt live upgrades, and existing live upgrade technology has a high risk of failure. The cost and timescales for the development of defense software are notorious; major systems typically require years to reach deployment, rather than the days or hours that will be essential in a future information war. 
The Eternal system attempts to address these problems by providing:

- Operation in heterogeneous hardware and software environments by use of the Common Object Request Broker Architecture (CORBA) distributed object computing standard [22], and unmodified commercialoff-the-shelf (COTS) hardware platforms, operating systems, and CORBA ORBs.

- Robust fault tolerance by use of object replication, so that if one replica is disabled by a fault then another replica can still continue to provide the required service. Both active and passive replication are provided; however, only active replication can achieve the rapid recovery from faults that is required in many defense systems.

- Live software upgrades by use of object replication, so that one replica can be upgraded while another replica continues to provide service. The live upgrade technology of Eternal is robust and requires no more skill of the application programmers than is required for programming the application.

- Reduced software development costs and timescales. With Eternal, an application programmer writes a standard CORBA program and that program is automatically rendered fault tolerant by Eternal. In current practice, the highly-specialized mechanisms for fault tolerance are inextricably mixed into the algorithms of the application program, greatly increasing the difficulty and complexity of programming the application. Moreover, the testing of fault tolerance is time consuming, and skimping on such testing can be catastrophic.

Application programmers must be experts in the application domain; they cannot also be expected to be experts in fault tolerance. It is more appropriate for experts in fault tolerance to program the fault tolerance mechanisms once only (as in the Eternal system), and then apply them to many defense applications. The application programming is simpler, with lower costs and shorter development timescales.

\section{The Eternal System}

The Eternal system is based on the Common Object Request Broker Architecture (CORBA) defined by the Object Management Group (OMG) [22]. CORBA provides modular distributed object programming, location transparency within a distributed system, portability of programs across platforms, and interoperability between diverse platforms.

Eternal extends CORBA with capabilities for fault tolerance and live upgrades of application objects. Eternal

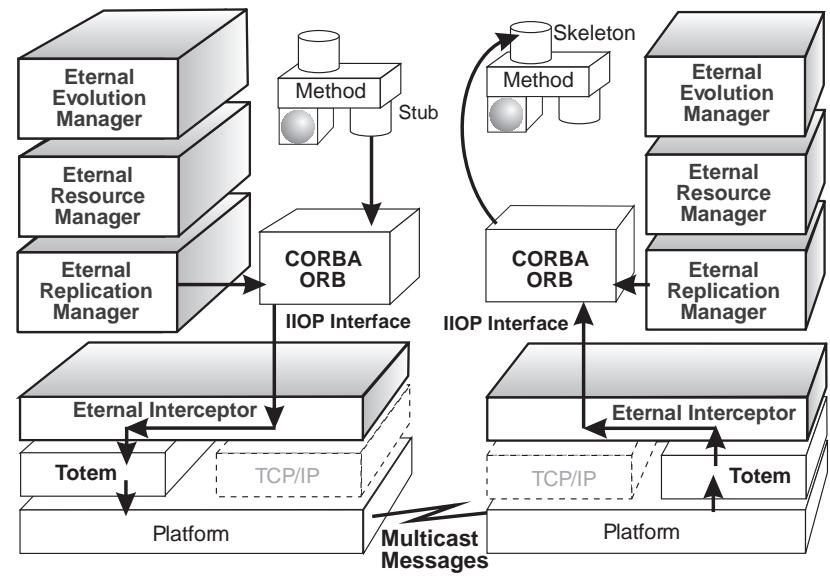

Figure 1: The Eternal system includes the Interceptor, Replication Manager, Evolution Manager and Resource Manager.

replicates objects, distributes the object replicas across the system and maintains consistency of the states of the replicas of an object. Both client and server objects can be replicated, and objects can act as both clients and servers.

In the Eternal system, the replicas of an object form an object group, a collection of objects typically located on different computers. An object can invoke methods on an object group (i.e., simultaneously on all of the members) in a transparent manner so that the invoker of the method is not aware of the type or degree of the replication, membership of the object group, or location of the members. A client object invokes the methods on a server object group as though the server were a single object.

The Eternal system includes the Interceptor, Replication Manager, Evolution Manager and Resource Manager, as shown in Figure 1. The CORBA Object Request Broker (ORB) packages the method invocations and responses into messages formatted according to the Internet Inter-ORB Protocol (IIOP), which are transmitted via TCP/IP. The Interceptor [17] captures the IIOP messages, and diverts them to the Replication Manager which, in turn, passes the messages to a multicast group communication system.

The group communication system multicasts the messages to the group and delivers the messages reliablly and in total order to all members of the group. Over local-area networks, multicast group communication protocols, such as Totem [13], are now as efficient as point-to-point communication using TCP/IP, so no performance disadvantage results from replacing TCP/IP by such a multicast protocol. For operation over the Internet, multicast group communication protocols, such as FTMP [15], are currently being developed.

The Replication Manager translates method invocations on an object group into method invocations on the in- 


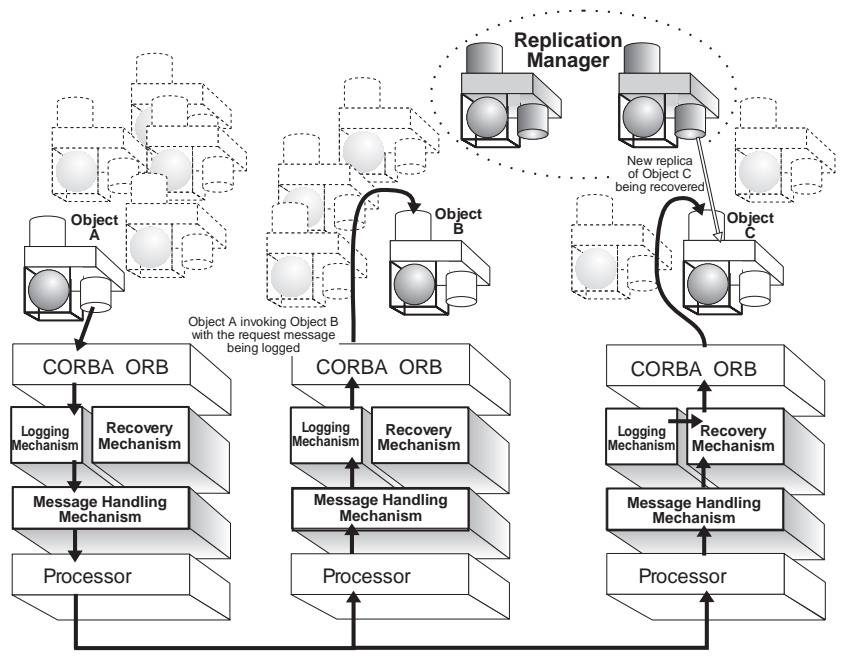

Figure 2: The Eternal Replication Manager, working in concert with the Message Handling, Logging and Recovery Mechanisms, provides object replication and maintains consistency of the states of the replicas. The mechanisms provide detection of duplicate invocations and duplicate responses, transfer of state between the object replicas, and consistent scheduling of concurrent operations.

dividual object replicas. To achieve replica consistency, the Replication Manager utilizes the reliable totally ordered message delivery of the group communication system. Using the Message Handling, Logging and Recovery Mechanisms, shown in Figure 2, the Replication Manager provides detection of duplicate invocations and duplicate responses, transfer of state between the object replicas, and consistent scheduling of concurrent operations.

The Evolution Manager performs the automated upgrade and evolution of application objects while they continue to execute. The replicas of an object can be stopped and replaced, one at a time, while other replicas continue to provide service. A sequence of replacements, each implementing a small modification to a single replica, can achieve substantial modifications without stopping the system. The Evolution Manager invokes the Replication Manager to create replicas of the new versions of the objects and to remove replicas of the old versions.

The Resource Manager determines appropriate allocations of objects to processors and distributes the replicas across the system. It monitors the behavior of the objects and the use of the resources, and can move objects and change the type or the number of replicas to meet performance objectives. To meet soft real-time deadlines, it uses a distributed least-laxity scheduling algorithm.

The Replication, Evolution and Resource Managers are themselves implemented as collections of CORBA objects, and can thereby benefit from CORBA's interoperability and Eternal's fault tolerance and live upgrade capabilities.

\section{Interception}

The Eternal Interceptor [17] is a non-ORB-level, nonapplication-level module that "attaches" itself to every executing application object, transparently to the application and the ORB, and is capable of modifying the object's behavior as desired.

Current operating systems provide hooks that can be exploited to develop modules such as interceptors. With the Unix operating system, there are at least two possible implementations of interceptors. The /proc-based implementation provides for interception at the level of system calls. The library interpositioning implementation provides for interception at the level of library routines. The techniques differ, but the intent and the use of interceptors is the same in both cases.

The specific system calls to intercept in a / proc-based implementation or the specific library routines to redefine in a library-interpositioning implementation, depends on the extent of the information that the interceptor must extract (from the ORB or the application) to enhance the application with new features. The interceptor may capture all, or a particular subset, of the system calls or library routines used by the application, depending on the feature being added. The Eternal Interceptor currently employs the library interpositioning implementation, because of its lower overheads and ease of deployment with various ORBs.

The Interceptor monitors the operating system calls made by the objects to establish IIOP connections over TCP/IP, and to communicate IIOP messages over those connections. The Interceptor catches the IIOP messages before they reach TCP/IP, and diverts them instead to the Replication Manager. The Replication Manager multicasts the messages to the object groups using the group communication system that delivers the messages to the object groups reliably and in total order. The interception approach of Eternal requires no modifications to the ORB, the operating system, or the application.

\section{Replication Management}

In Eternal, the replicas of an object form an object group. Communication occurs between client and server object groups, rather than between individual client and server objects. Each object group has a unique object group identifier.

A reliable totally ordered multicast group communication system is used to communicate invocations and responses between client and server object groups. This ensures that all of the replicas of an object receive the same sequence of messages in the same order, which facilitates replica consistency.

In Eternal, a Logging Mechanism on each processor is responsible for recording invocations, responses and check- 
points of the replicas hosted on that processor. Typically, each processor hosts many different object groups. Thus, the Logging Mechanism maintains a single physical log for the processor, and the $\log$ is indexed by the object group identifier.

The $\log$ is a sequence of $\log$ records, each containing both an IIOP message (or a checkpoint represented as an IIOP message) and a special Eternal-specific header associated with the IIOP message for duplicate detection, garbage collection of the log, etc. The records are stored in the $\log$ as they are delivered reliably and in total order to the Logging Mechanism by the underlying multicast group communication system. The log contains only nonduplicate records.

For all outgoing messages from the replicas that it manages, the Replication Manager receives the IIOP message from the Interceptor, and passes it to the Message Handling and Logging Mechanisms, which record the message header for duplicate detection and then encapsulates the message in an Eternal-specific header and passes it to the underlying multicast group communication system for transmission.

For all incoming messages to the replicas that it manages, the Replication Manager receives the encapsulated IIOP messages from the underlying multicast group communication system and routes the encapsulated messages with the Eternal-specific header to the Message Handling and Logging Mechanisms. The Logging Mechanism determines whether the message is a duplicate and records its header to allow detection of future duplicates. For passively replicated object groups, the Logging Mechanism logs the entire message for possible use during recovery. The Message Handling Mechanism uses the header to determine the target group and local replica for the message and delivers the message to the application.

For every replicated CORBA object that it manages, the Replication Manager also receives group membership change notifications from the underlying multicast group communication system. Thus, the Replication Manager is aware of the addition of replicas to, or the removal of replicas from, the object groups that it manages. When a new or recovering or upgraded replica is introduced, the Replication Manager initiates the transfer of state to the new replica by invoking the Logging Mechanism.

\section{Replica Consistency}

To ensure that the states of the replicas of an object are updated consistently, Eternal exploits the reliable totally ordered message delivery service of an underlying multicast group communication system that provides virtual synchrony guarantees. The replicas start in the same initial state. The method invocations, and the corresponding responses, are contained in multicast messages. The multicast messages are delivered in the same total order at each of the object replicas. Consequently, each of the object replicas performs the operations in the same order, and the states of the object replicas remain consistent.

In addition to the reliable totally ordered multicast group communication system, Eternal employs the following mechanisms to maintain replica consistency:

- Transfer of state between replicas, ensuring that all of the replicas agree on which operations precede the state transfer and which follow it,

- Detection of duplicate invocations and duplicate responses that are generated by two or more replicas of an object, and

- Consistent scheduling of multithreaded concurrent operations.

\subsection{State Transfer}

Every replicated object can be regarded as having three kinds of state: application state, programmed into the object by the application programmer, ORB state, maintained by the ORB for the object, and infrastructure state, invisible to the application programmer and maintained by Eternal for the object. Application state is typically represented by the values of the data structures of the object. ORB state is vendor-dependent and consists of the values of the data structures (last-seen request identifier, threading policy, etc). Logging state is independent of, and invisible to, the object as well as to the ORB, and involves only information that is needed to maintain replica consistency.

The Logging and Recovery Mechanisms of Eternal ensure that all of the replicas of an object are consistent in application, ORB and infrastructure state. State transfer to a new or recovering or upgraded replica includes the transfer of application state to the replica, ORB state to the ORB hosting the new replica, and infrastructure state to the Message Handling and Logging Mechanisms for the replica.

In order that application state can be transferred from one replica to another, every object that is replicated must inherit the Checkpointable interface. This interface contains a get_state() method for retrieving an object's state and a set_state() method for assigning an object's state. The frequency of checkpointing is determined as a property by the application deployer for each object group individually.

For a passively replicated object group (see Section 6.2), the Recovery Mechanism transfers the state of the primary replica to the backup replicas periodically. For an actively replicated object group, the Recovery Mechanism transfers state only to activate a new or recovering or upgraded replica. In both cases, the Recovery Mechanism fabricates an IIOP message for get_state() to dispatch to the object 
group to obtain the application state from one of the existing replicas. The Recovery Mechanism at the existing replica(s) piggybacks the ORB state and infrastructure state for the existing replica(s) onto the application state returned in the response message for the invocation of get_state(). The response message is then transmitted to the backup replica(s) or the new or recovering or upgraded replica, and is logged at those replicas.

The Recovery Mechanism at a new or recovering or upgraded replica, whether actively or passively replicated, extracts that message from the log, strips off the infrastructure state and the ORB state, and uses it to initialize the infrastructure state and the ORB state for that replica. The application state (the return value of get_state()) is passed by the Recovery Mechanism as an argument to set_state(), which it invokes on the new or recovering or upgraded replica.

All of the incoming invocations and responses must be enqueued until the set_state() invocation is delivered. This queue also contains the get_state() invocation dispatched earlier to the object group; all of the invocations and responses prior to this invocation are discarded from the queue. The get_state() and set_state() invocations must appear to occur at the same logical point in time because the return value of the get_state() is the parameter of the set_state().

Thus, when the set_state() invocation is received by the Recovery Mechanism at a new or recovering or upgraded replica, the invocation moves to the head of the incoming message queue (a position previously occupied by the get_state() message), and is delivered to the new or recovering or upgraded replica. The remaining enqueued messages are applied after the state transfer. The logged get_state() invocation is never applied to the new or recovering or upgraded replica; it simply serves to represent the synchronization point, in the totally ordered message sequence, at which the state transfer must occur through its corresponding set_state() message.

\subsection{Duplicate Detection and Suppression}

The Message Handling Mechanism matches up responses with their corresponding invocations, and detects and suppresses duplicate invocations, responses and state transfer messages. To be able to match incoming response messages with their corresponding invocations, the Message Handling Mechanism inserts an invocation (response) identifier into the Eternal-specific header for each outgoing IIOP invocation (response) message. A part of the invocation identifier, the operation identifier, uniquely represents the operation consisting of the invocation-response pair. All replicas in an object group choose the same operation identifier, which is included in both invocation and response messages.
The Message Handling Mechanism records, for each source group on its processor, the invocation identifiers of all outgoing invocations for which responses are expected. When a response arrives, the Message Handling Mechanism delivers the response only if the operation identifier in the received response identifier corresponds to the operation identifier in the invocation identifier of an outstanding invocation.

Operation identifiers are also used to discard duplicate invocations and duplicate responses, so that only nonduplicate messages are delivered to the destination group. Each destination group corresponds to one or more source groups. To enable duplicate detection, for each source group that sends messages to a destination group, the Message Handling Mechanism at a destination processor records the operation identifier associated with the lastreceived message from the source group.

The list of operation identifiers for the outstanding invocations for which the existing replicas are awaiting responses is part of the infrastructure state that the Logging Mechanism stores and manages. The infrastructure state also contains information that the Logging Mechanism uses for duplicate detection and garbage collection of the log, including the list of last-seen operation identifiers from every sender group.

For every outgoing IIOP message that it receives from the Replication Manager, the Message Handling Mechanism inserts, but does not record, a unique operation identifer into the Eternal-specific header of the encapsulated message. For every incoming encapsulated IIOP message, the Message Handling Mechanism uses the information in the Eternal-specific header to detect and suppress duplicate messages, and passes only non-duplicate messages (along with sufficient information about the target object group) to the Replication Manager for delivery to the application.

\subsection{Multithreading}

Many commercial ORBs are multithreaded, and multithreading can yield substantial performance advantages. Unfortunately, the specification of multithreading in the CORBA standard does not place any guarantee on the order of operations dispatched by a multithreaded ORB. In particular, the specification of the Portable Object Adapter (POA), which is a key component of the CORBA standard, provides no guarantee about how the ORB or the POA dispatches requests across threads. The ORB may dispatch several requests for the same object within multiple threads at the same time.

In addition to ORB-level threads, the CORBA application itself can be multithreaded, with the thread scheduling determined by the application programmer. The application programmer must ensure correct sequencing of operations 
and must prevent thread hazards. Careful application programming can ensure thread-safe operations within a single replica of an object; however, it does not guarantee that threads and operations are dispatched in the same order across all of the replicas of an object. The application programmer should not need to be responsible for concurrency control and ordering of dispatched operations in replicated objects to provide strong replica consistency.

To maintain strong replica consistency for multithreaded objects, the Eternal system enforces deterministic behavior across all of the replicas of a multithreaded object by controlling the dispatching of threads and operations identically within every replica through a deterministic operation scheduler.

The operation scheduler dictates the creation, activation, deactivation and destruction of threads within every replica of a multithreaded object, as required for the execution of the current operation "holding" the logical threadof-control. Exploiting the thread library interpositioning mechanisms of Eternal's Interceptor, the scheduler can override any thread or operation scheduling that either the multithreaded ORB, or the replica itself, performs.

Based on the incoming reliable totally ordered message sequence, the scheduler at each replica decides on the immediate delivery, or the delayed delivery, of the messages to that replica. At all of the replicas, the schedulers' decisions are the same and, thus, operations and threads are dispatched in the same order at all of the replicas of the multithreaded object.

A more complete description of the mechanisms that Eternal uses for state transfer, duplicate detection and suppression, and multithreading can be found in $[14,18,19]$.

\section{Fault Tolerance}

\subsection{Fault Model}

Eternal protects against only omission faults, or against both omission faults and commission faults. These types of faults are broadly defined as follows:

- An omission fault occurs when an object or processor sends no further messages, i.e., it crashes, or omits to send an expected message but does send subsequent messages.

- A commission fault occurs when an object or processor sends a message that is syntactically or semantically incorrect, such as a mutant message. Mutant messages are two or more messages that purport to be the same message but that have different contents.

\subsection{Types of Replication}

The application and the types of faults that must be tolerated dictate the types of replication that are employed.

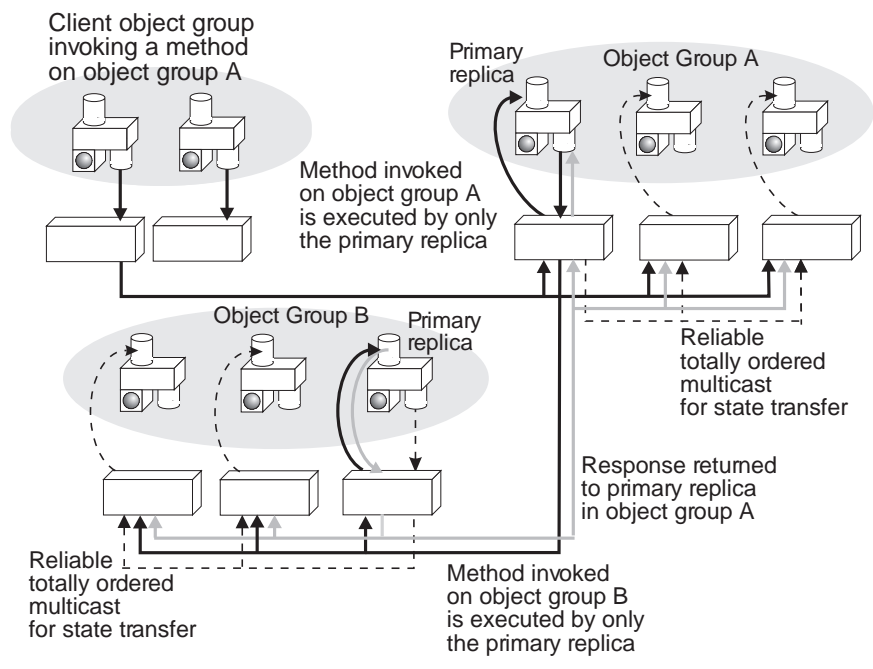

Figure 3: Passive replication. The primary replica in the object group executes the method and the Recovery Mechanism transfers the state of the primary to the nonprimary replicas at the end of the method invocation.

To protect against omission faults, Eternal uses the standard techniques of passive replication and active replication (without majority voting). To protect against commission faults, Eternal uses active replication with majority voting together with a more robust underlying group communication protocol, such as SecureRing [10], which substantially increases the cost of replication and fault tolerance.

\subsubsection{Passive Replication}

In passive replication, when a client object invokes a method on a server object group, Eternal multicasts the invocation to the server object group and only one of the server replicas, the primary replica, executes the method, as shown in Figure 3. The Replication Manager at each of the other replicas retains the message containing the method invocation so that those replicas can execute the method, if the primary replica fails. At the end of the method execution, Eternal multicasts the updated state of the primary replica to the nonprimary replicas and multicasts the results to the invoking object. The state transferred to the nonprimary replicas serves as a checkpoint to which the state can be rolled back, if the primary replica fails. During the execution of the method, the states of the nonprimary replicas may differ from that of the primary replica; however, the state transfer achieves replica consistency at the end of the method execution. The underlying reliable totally ordered multicast protocol ensures that either all of the nonprimary replicas have the updated state of the object or, alternatively, none of them has the updated state. 


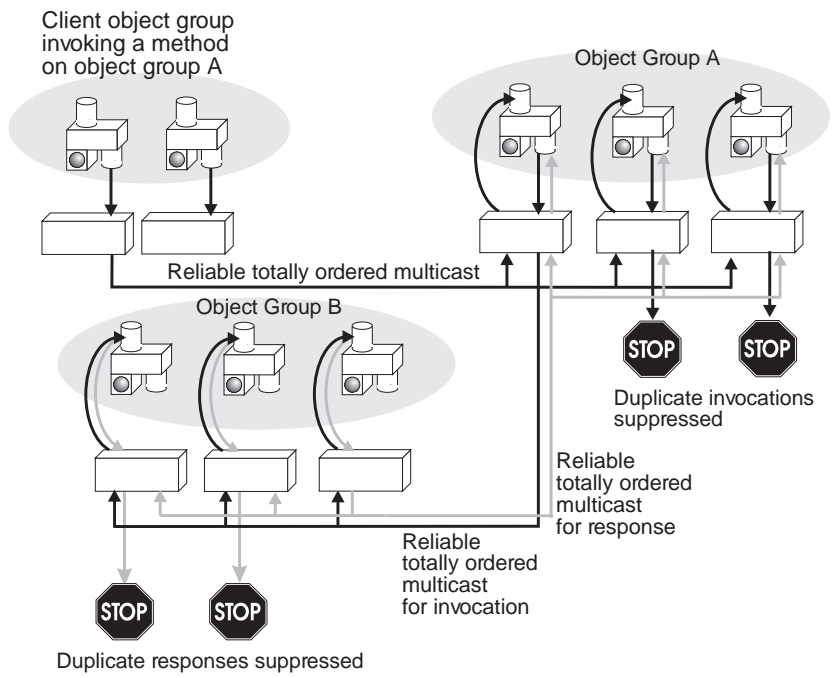

Figure 4: Active replication. All of the replicas within the object group execute the method. Duplicate invocations and duplicate responses are detected and suppressed by the Message Handling Mechanism.

In Figure 3, object groups $A$ and $B$ each contain three passive replicas. A client object invokes a method of object group $A$, and only the primary replica in object group $A$ executes the method. That method invocation results in the invocation of a further method on object group $B$, and again, only the primary replica in object group $B$ executes the method. When the primary replica in object group $B$ has completed the method execution, the Recovery Manager transfers the state of that replica to the nonprimary replicas in object group $B$ and returns the results to the replicas in object group $A$.

\subsubsection{Active Replication}

In active replication, when a client object invokes a method on a server object group, Eternal multicasts the method invocation to the server object group and each of the server replicas then executes the method, as shown in Figure 4. The underlying reliable totally ordered multicast protocol ensures that all of the replicas of an object receive the same messages in the same order, and that they can thus execute the methods in the same order. This ordering of method invocations and responses ensures that the states of the replicas are consistent at the end of each operation.

In Figure 4, object groups $A$ and $B$ each contain three active replicas. A client object invokes a method of an object group $A$, which in turn invokes a method of an object group $B$. Each of the replicas in object group $B$ executes the method invocation, and Eternal multicasts the results at the end of the execution and, similarly, for each of the replicas in object group $A$.

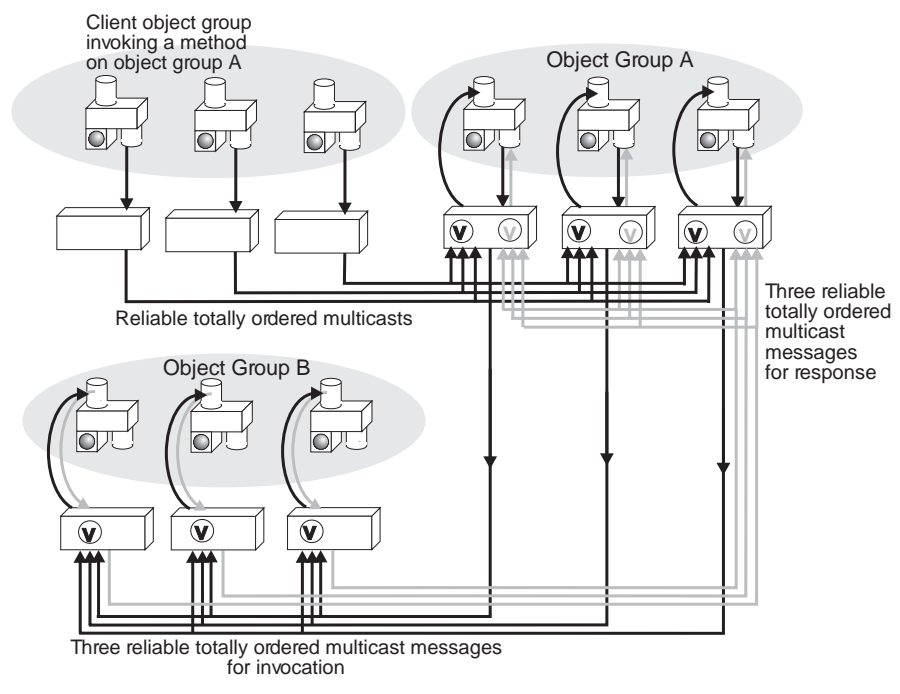

Figure 5: Active replication with majority voting. Using a Voting Mechanism, the Replication Manager subjects the invocations (responses) to majority voting in order to produce a single invocation (response).

\subsubsection{Active Replication with Majority Voting}

To tolerate both omission and commission faults, active replication with majority voting must be used, as shown in Figure 5. In the past, majority voting was used in synchronous systems for safety-critical applications such as aircraft flight control [28]. Multicast group communication systems that provide reliable totally ordered message delivery in a model of virtual synchrony make majority voting possible in asynchronous systems. Most group communication systems tolerate only crash faults. A more robust group communication system, such as SecureRing [10], must be used to tolerate arbitrary faults.

Majority voting requires at least three-way active replication. In an environment that is subject to arbitrary faults, the invocation (response) first received might be erroneous and, thus, duplicate invocations (responses) cannot be suppressed. Rather, to tolerate arbitrary faults, the invocations (responses) from different replicas in the same object group are collected at the invokee (invoker) and combined using majority voting to produce a single invocation (response).

In Figure 5, object groups $A$ and $B$ each contain three active replicas and majority voting is used. A client object invokes a method of object group $A$, which in turn invokes a method of object group $B$. Using a Voting Mechanism, the Replication Manager subjects the invocations to majority voting in order to produce a single invocation. Then, each of the replicas in object group $A$ executes the method, and Eternal multicasts the results, and similarly for each of the replicas in object group $B$. Using the Voting Mechanism, 
the Replication Manager likewise subjects the responses to majority voting in order to produce a single response.

More details about the mechanisms needed to tolerate both omission faults and communications faults can be found in [16]

\subsection{Fault Detection and Recovery}

Detection of faults in Eternal (and Totem) is based on unreliable fault detectors because, in an asynchronous distributed system that is subject to faults, it is impossible to distinguish between an object or processor that has failed and one that is merely slow. However, if commission faults must also be tolerated, then a more robust fault detector is required [9].

Recovery from faults requires more care for passive replication than for active replication. For an object that is passively replicated, the effect of a fault depends on whether the failed replica is a primary or nonprimary replica. If a nonprimary replica fails during the execution of a method, the Replication Manager removes it from the group, while the primary replica continues to execute the method. Thus, the failure of a nonprimary replica is transparent to the client object that invoked the method.

To consider the failure of a primary replica, envisage a passively replicated object with several replicas, each on a different processor. A method invoked on an object group containing those replicas is multicast to both the primary and nonprimary replicas. The nonprimary replicas do not execute the method; rather, the Logging Mechanism for a nonprimary replica logs the method invocation until it receives the response from the primary replica. If the primary replica fails, the Replication Manager determines a new primary replica. The new primary replica must execute all of the methods for which it has not received a response from the prior primary replica. Invocations on other objects, and even responses, can be generated by both the original replica and the new primary replica. The Message Handling Mechanism suppresses duplicate invocations (responses) that occur after the fault.

For active replication, if any one of the server replicas fails, or is removed for upgrading during the execution of a method, service is not interrupted (because the method is executed by the other server replicas) and the results are returned to the client object. The server replicas also preserve the state of the server object for subsequent transfer to a new or recovered or upgraded server replica. To sustain operation, at least one server replica must be operational and, to tolerate a fault, two or more server replicas are needed.

\section{Evolution Management}

Without the ability to upgrade the software, and also the hardware, no application can claim to be able to operate continuously. Exploiting object replication, the Eternal Evolution Manager readily supports evolution of, and upgrades to, the hardware. Hardware components can fail, be repaired or replaced, and be reintegrated into the system without interruption of service. If the replacement hardware is of a different design (e.g., different byte order or data representation), the interoperability features of CORBA enable the application to adapt to the change.

With existing technologies, in a conventional application, the system must be stopped in order to upgrade the software (i.e., the application objects themselves). With Eternal, the system need not be stopped to upgrade the application objects. By exploiting object replication, the Evolution Manager accomplishes the overall change to the objects incrementally and systematically, while the application continues to operate.

The Evolution Manager, which comprises the Preparer and the Upgrader shown in Figure 6, performs the upgrade of a large program in a sequence of steps. Each step of the sequence is completed and demonstrated to operate satisfactorily before the next step is undertaken. A step in the sequence consists of three phases:

- A preparation phase in which the programmer prepares a new upgraded program that is to replace the existing program

- A preliminary preprocessing phase that involves the Preparer with the assistance of the human

- A fully automatic upgrade phase that involves the Upgrader.

\subsection{The Preparer}

To upgrade an object class, the programmer submits, to the Preparer, the code of both the existing object class and a new version of the class. The Preparer compares the two classes and determines the differences. With assistance from the programmer, the Preparer generates one or more intermediate classes to facilitate the upgrade, and compiles and deposits those classes into the CORBA Implementation Repository for use by the Upgrader. No special skill is required of the application programmer beyond that required to program the application program being upgraded.

\subsection{The Upgrader}

The Upgrader upgrades an object of a class using a sequence of invisible upgrades, each of which moves closer to the desired overall upgrade. The actual upgrade is performed in a single atomic action when all program code and data structures are in place. Further invisible upgrades then remove obsolete or transitional code. The Upgrader ensures that at least one replica of an object continues to provide service while another replica is being upgraded. 


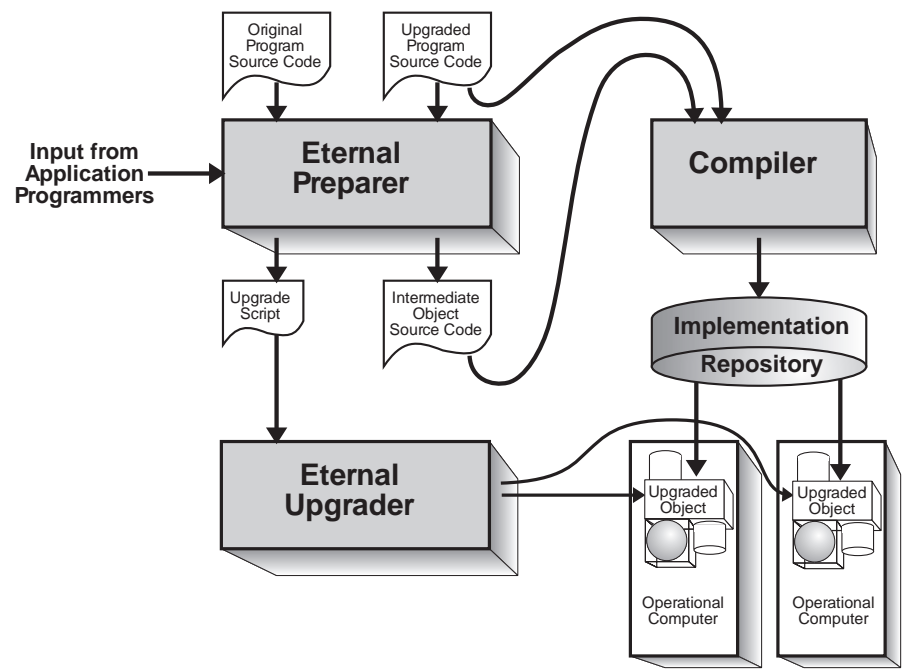

Figure 6: The Eternal Evolution Manager, consisting of the Preparer and the Upgrader, exploits object replication to achieve hardware and software upgrades without stopping the system.

Particular care is required for upgrades that modify the attributes (local state variables) of an object. Code must be generated by the Preparer with the assistance of the programmer, and invoked by the Upgrader, to set appropriate values for new attributes. Even more care is required if the signatures (parameters and their types) of the methods of the objects are changed. Several classes of objects may need to be upgraded together in a coordinated upgrade sequence.

If evolution is required but fault tolerance is not, then replication is necessary only while the system is being upgraded. Such systems operate normally with unreplicated objects, but additional replicas are introduced when needed to support live upgrade and evolution. Replication thus provides not only fault tolerance but also live upgrades that allow distributed object applications to grow and evolve without interruption of service.

\section{Resource Management}

Typical distributed object applications, particularly defense applications, are very complex and are incompletely understood, and it is difficult for the application programmer to obtain accurate projections of resource requirements and behavioral characteristics. Consequently, resource management in Eternal is implemented as a Resource Manager for the entire system and a Profiler and a Scheduler for each processor, as shown in Figure 7. Logically, there is only a single copy of the Resource Manager, although it may be replicated for fault tolerance. Each Profiler and each Scheduler, however, is specific to an individual processor and is interfaced to both the ORB and the operating system.

\subsection{The Resource Manager}

The Resource Manager allocates the object replicas to the processors based on the current loads on the processors, and moves objects from one processor to another. As new tasks are introduced into the system, the Resource Manager decides whether the available resources can satisfy the requests and allocates the resources accordingly. During operation, the Resource Manager might determine that a resource is overloaded or that a task is not meeting its deadlines, necessitating reallocation of the object replicas. If a processor is lost because of a fault, the Resource Manager might need to reallocate the object replicas to maintain a sufficient degree of replication to satisfy fault tolerance requirements.

For each application task, the Resource Manager maintains a list of the method invocations required for that task, a deadline for completion of the task, and an importance metric that is used to decide which tasks should be abandoned if a system overload occurs.

For each method of each object, the Resource Manager maintains estimates of the processing and communication times for invocations of that method. These estimates are used to determine the initial laxity of the application task when it starts to execute.

The Resource Manager works in concert with the Profilers and Schedulers on the processors. The Profilers monitor the behavior of the application objects and measure the current load on the processors' resources. The Resource Manager allocates objects to processors, the objects execute and use resources, and the Profilers report resource usage to the Resource Manager. The Resource Manager can determine the degree of replication of each application object and can reallocate resources and reschedule objects on the processors, to maximize the system utility and to maintain a uniform load on the resources. The Schedulers exploit information collected by the Resource Manager to schedule the tasks to meet soft real-time deadlines.

The Resource Manager employs a three-level feedback loop. The tightest level (milliseconds) uses measurements of elapsed time to refine the estimated residual laxity of executing tasks, which are used for least-laxity scheduling. The second level (fractions of a second) uses measurements of elapsed time and measurements of the resource load to refine the initial estimates of the laxity for the tasks as they start. The third level (several seconds) uses the measured resource load and residual laxities to revise the allocation of objects to processors.

\subsection{The Profilers}

The Profilers monitor the current load on the processors and, therefore, can detect significant deviations in performance. They supply feedback to the Resource Manager, which determines the allocation of the objects to the processors. 


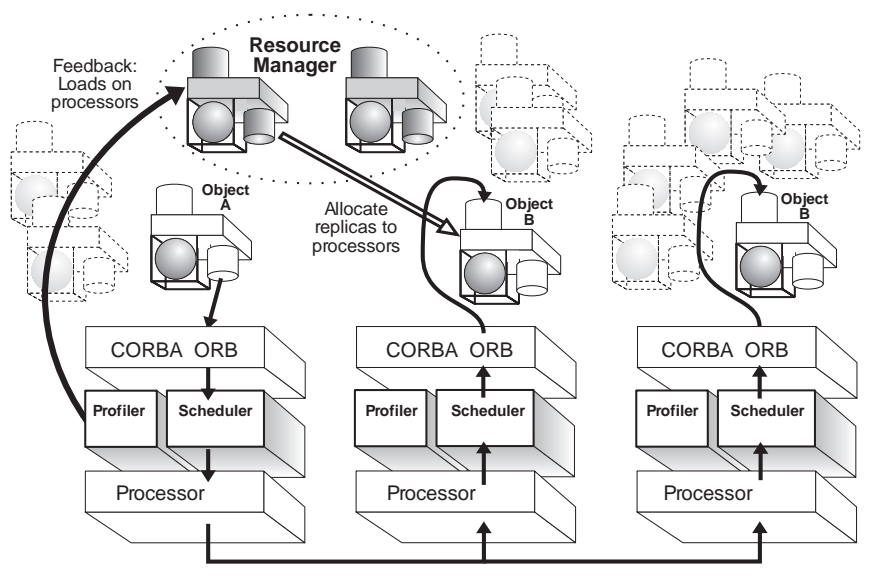

Figure 7: The Eternal Resource Manager, working in concert with the Profilers and the Schedulers on each of the processors, employs object migration algorithms to balance the loads on the processors and also a distributed least-laxity scheduling algorithm to schedule the tasks.

Each Profiler is specific to an individual processor and reports, for each resource associated with its processor the current utilization of the resource, which determines whether a resource is overloaded and whether to reallocate objects to other processors to balance the load or to decrease their degrees of replication.

Each Profiler also monitors the behavior of the application objects located on its processor and reports, for each application object, the time required for the object to execute on the processor and the proportion of each resource allocated to the object.

\subsection{The Schedulers}

The Schedulers employ a distributed least-laxity scheduling algorithm, which is effective, provided that the processors are not overloaded. The processor utilizations are assumed to provide sufficient margins to accommodate statistical fluctuations in the load with acceptable probability.

In least laxity scheduling, the laxity of task $t$ represents a measure of urgency of the task. The laxity is defined by:

$$
\text { Laxity }_{t}=\text { Deadline }_{t}-\text { Projected_latency }_{t}
$$

where Deadline Dis $_{t}$ the time by which task $t$ must be completed and Projected_latency $y_{t}$ is the estimated time to complete task $t$.

A more complete description of the resource management strategies employed by the Eternal system can be found in $[6,7,12]$.

\section{Prototype Implementation}

Our prototype implementation of the Eternal system operates using unmodified commercial ORBs, including Inprise's VisiBroker, Iona's Orbix, Xerox PARC's ILU,
Object-Oriented Concept's ORBacus and Washington University's real-time TAO ORB. The implementation is designed for Solaris 2.6 but also operates on RedHat Linux. A port to WindowsNT is in progress.

The current implementation exploits library interpositioning, which is less dependent on operating system specific mechanisms and has lower overheads than our initial implementation, which was based on intercepting the / proc interface of the Solaris operating system. Either approach (library interpositioning or using / proc) allows Eternal to be used with diverse commercial ORBs, with no modification to either the ORB or the application. The vendor's implementation of CORBA must, of course, support IIOP, as the CORBA standard mandates.

The overheads of Eternal are in the range of $10-15 \%$ for remote invocations/responses with triplicated clients and triplicated servers. These low overheads include the cost of interception and replication, as well as that of multicasting GIOP messages using the Totem multicast group communication system [13].

For example, using Sun UltraSPARC2 167 and 200 $\mathrm{MHz}$ workstations and $100 \mathrm{Mbit} / \mathrm{s}$ Ethernet, a remote invocation and response with an unreplicated client and an unreplicated server running over the VisiBroker ORB, without Eternal, required $0.330 \mathrm{~ms}$. In this case, the client and server communicate using IIOP messages transmitted over TCP/IP.

Using Eternal, for the same platform and application, with three-way actively replicated client and server objects running over VisiBroker, a remote invocation and response required $0.369 \mathrm{~ms}$, which represents an overhead of $12 \%$ over the unreplicated case. These measurements involved an actively replicated client object repeatedly invoking an actively replicated server object using deferred synchronous communication without message packing.

With three-way passively replicated clients and servers, a remote invocation and response required $0.374 \mathrm{~ms}$, which represents an overhead of $15 \%$ over the unreplicated case. These measurements involved a passively replicated client object, with the primary client replica repeatedly invoking the passively replicated server object. The state transfers for both client and server objects were hand-coded.

\section{Related Work}

Several systems that extend CORBA with object replication and fault tolerance have been developed.

The Electra toolkit implemented on top of Horus provides support for fault tolerance by replicating CORBA objects, as does Orbix+Isis on top of Isis [1, 11]. Unlike Eternal, Electra and Orbix+Isis are non-hierarchical object systems that support only active replication. Both Electra and Orbix+Isis use an integration approach in that 
the replication and group communication mechanisms are integrated into the ORB and require modification to the ORB. In contrast, Eternal uses the interception approach, which requires no modification to the ORB.

Another approach to fault tolerance, adopted by the OpenDREAMS toolkit [4], adds replication and group communication as services (implemented as CORBA objects) on top of the ORB, and requires no modification to the ORB. The service approach exposes the replication of objects to the application programmer and allows the programmer to modify the class library to construct customized replication and group communication services. In contrast, Eternal is transparent to the application and the application programmer.

The Distributed Object-Oriented Reliable Service (DOORS) [24] adds support for fault tolerance to CORBA applications by providing replica management and fault detection as service objects above the ORB. DOORS supports passive but not active replication and is not based on multicast group communication, as is the Eternal system. The DoorMan management interface monitors DOORS and the underlying system to fine-tune the functioning of DOORS and to take corrective action, if their hosts are suspected of being faulty.

The Maestro toolkit [27] adds reliability and high availability to CORBA applications in settings where it is not feasible to make modifications at the client side. It includes an IIOP-conformant ORB with an open architecture that supports multiple execution styles and request processing policies. The replicated updates execution style can be used to add reliability and high availability on the client side.

The AQuA framework [3] employs the Ensemble/Maestro [26, 27] toolkits, the Proteus dependability property manager, and the Quality of Service for CORBA Objects $(\mathrm{QuO})$ runtime system. Proteus determines the type of faults to tolerate, the replication policy, the degree of replication, the type of voting to use and the location of the replicas. Using a Quality of Service Description Language (QDL) to specify an application's expected usage patterns and QoS requirements, QuO modifies the configuration to meet those requirements dynamically, and provides mechanisms for measuring and enforcing Quality of Service contracts and taking appropriate actions when those contracts are violated.

Another CORBA-based system that provides adaptation to dynamic and unpredictable changes in the computing environment has been developed by Nett, Gergeleit and Mock [20]. Like Eternal, their system uses integrated monitoring, dynamic execution time prediction, and scheduling to provide time-awareness for standard CORBA object invocations.

Shokri, Hecht, Crane, Dussault and Kim [25] make the case that effective fault handling in complex distributed applications requires the ability to adapt resource allocation and fault tolerance policies dynamically in response to changes in the environment, application requirements and available resources. The Eternal system supports this viewpoint in its implementation of fault tolerance and resource management.

While the above systems provide support for fault tolerance and resource management, they do not provide support for live upgrade and evolution of objects.

The Simplex Architecture [5], which is intended for online upgrades of control systems, is based on two abstractions, the replaceable unit abstraction and the cell abstraction. The replaceable unit abstraction allows an existing software module to be replaced online by another module with similar or enhanced functionality, while the cell abstraction represents a protected module which cannot be affected by other modules. These abstractions have been implemented in a real-time POSIX testbed, based on publish/subscribe communication, which is quite different from the multicast group communication employed by the Eternal system.

Another system that supports upgrades of system software, hardware and application software has been developed by Kanevsky, Krupp and Wallace [8]. Their system shares many characteristics with the Simplex Architecture but differs in its application to the evolution of long lifecycle defense systems. In particular, they have applied their technology to the multiple target tracking part of a surveillance radar system.

Neither of those two systems has addressed the general problem of live upgrades of object-oriented programs that the Eternal system addresses.

\section{Conclusion}

Defense applications of the future will be complex distributed object applications that must operate continuously without ever stopping. Those applications will be difficult enough to develop without the additional programming required to provide fault tolerance and live upgrades.

By replicating CORBA objects and maintaining strong replica consistency, the Eternal system provides support for fault tolerance and live upgrades of defense applications. Eternal simplifies the programming of those applications by exploiting the location transparency, portability and interoperability that CORBA provides and hiding the difficult issues of replication, consistency and recovery from the application programmer.

The prototype implementation of the Eternal system works with unmodified commercial-off-the-shelf CORBA ORBs, with overheads for triply replicated applications in the range of $10-15 \%$, compared to their unreplicated counterparts. These low overheads include the cost of 
interception and replication, as well as that of multicasting messages.

During the past year, we have been working with the Object Management Group to establish a standard for fault tolerance for CORBA [21, 23]. Currently, we are adapting the technology of the Eternal system to meet the requirements of that standard and to meet the needs of commercial CORBA applications.

\section{References}

[1] K.P. Birman and R. van Renesse, Reliable Distributed Computing with the Isis Toolkit, IEEE Computer Society Press, Los Alamitos, CA (1994).

[2] D. H. Craft, “'A study of pickling,' Journal of ObjectOriented Programming, vol. 5, no. 8, SIGS Publications, New York (January 1993), pp. 54-66.

[3] M. Cukier, J. Ren, C. Sabnis, W. H. Sanders, D. E. Bakken, M. E. Berman, D. A. Karr and R. E. Schantz, "AQuA: An adaptive architecture that provides dependable distributed objects,' Proceedings of the IEEE 17th Symposium on Reliable Distributed Systems, West Lafayette, IN (October 1998), pp. 245-253.

[4] P. Felber, B. Garbinato and R. Guerraoui, "Designing a CORBA group communication service," Proceedings of the IEEE 15th Symposium on Reliable Distributed Systems, Niagara on the Lake, Canada (October 1996), pp. 150-159.

[5] M. Gagliardi, R. Rajkumar and L. Sha, “Designing for evolvability: Building blocks for evolvable real-time systems,' Proceedings of the IEEE 1996 Real-Time Technology and Applications Symposium, Brookline, MA (June 1996), pp. 100-109.

[6] V. Kalogeraki, L. E. Moser and P. M. Melliar-Smith, "Dynamic modeling of replicated objects for dependable soft real-time distributed object systems,', Proceedings of the IEEE 4th Workshop on ObjectOriented Real-time Dependable Systems, Santa Barbara, CA (January 1999), pp. 48-55.

[7] V. Kalogeraki, P. M. Melliar-Smith and L. E. Moser " Using multiple feedback loops for object profiling, scheduling and migration in soft real-time distributed object systems,' Proceedings of the IEEE 2nd International Symposium on Object-Oriented Real-Time Distributed Computing, Saint Malo, France (May 1999), pp. 291-300.

[8] A. Kanevsky, P. C. Krupp and P. J. Wallace, "Paradigm for building robust real-time distributed mission-critical systems,', Proceedings of the IEEE
1995 Real-Time Technology and Applications Symposium, Chicago, IL (May 1995), pp. 33-40.

[9] K. P. Kihlstrom, L. E. Moser and P. M. Melliar-Smith, "Solving consensus in a Byzantine environment using an unreliable fault detector,' Proceedings of the International Conference on Principles of Distributed Systems, Picardie, France (December 1997), pp. 61-75.

[10] K. P. Kihlstrom, L. E. Moser and P. M. Melliar-Smith, "The SecureRing protocol for securing group communication,' Proceedings of the IEEE 31st Hawaii International Conference on System Sciences, vol. 3, Kona, HI (January 1998), pp. 317-326.

[11] S. Landis and S. Maffeis, "Building reliable distributed systems with CORBA,' Theory and Practice of Object Systems, vol. 3, no. 1 (April 1997), pp. 31-43.

[12] P. M. Melliar-Smith, L. E. Moser, V. Kalogeraki and P. Narasimhan "The Realize middleware for replication and resource management," Proceedings of the IFIP International Conference on Distributed Systems Platforms and Open Distributed Processing, Middleware '98, The Lake District, England (September 1998), pp. 123-138.

[13] L. E. Moser, P. M. Melliar-Smith, D. A. Agarwal, R. K. Budhia and C. A. Lingley-Papadopoulos, "Totem: A fault-tolerant multicast group communication system,' Communications of the ACM, vol. 39, no. 4 (April 1996), pp. 54-63.

[14] L. E. Moser, P. M. Melliar-Smith and P. Narasimhan, "Consistent object replication in the Eternal system," Theory and Practice of Object Systems, vol. 4, no. 2 (1998), pp. 81-92.

[15] L. E. Moser, P. M. Melliar-Smith, R. R. Koch and K. Berket, "A group communication protocol for CORBA,' Proceedings of the 1999 ICPP International Workshop on Group Communication, Aizu, Japan (September 1999), pp. 30-36.

[16] P. Narasimhan, K. P. Kihlstrom, L. E. Moser and P. M. Melliar-Smith, "Providing support for survivable CORBA applications with the Immune system,' Proceedings of the IEEE 19th International Conference on Distributed Computing Systems (May/June 1999), Austin, TX, pp. 507-516.

[17] P. Narasimhan, L. E. Moser and P. M. MelliarSmith, "Using interceptors to enhance CORBA," IEEE Computer, vol. 32, no. 7 (July 1999), pp. 62-68. 
[18] P. Narasimhan, L. E. Moser and P. M. Melliar-Smith "Replication and recovery mechanisms for strong replica consistency in reliable distributed systems,', Proceedings of the ISSAT 5th International Conference on Reliability and Quality in Design, Las Vegas, NV (August 1999), pp. 26-31.

[19] P. Narasimhan, L. E. Moser and P. M. Melliar-Smith, " Enforcing determinism for the consistent replication of multithreaded CORBA applications,', Proceedings of the IEEE 18th Symposium on Reliable Distributed Systems, Lausanne, Switzerland (October 1999), pp. 263-273.

[20] E. Nett, M. Gergeleit and M. Mock, “'An adaptive approach to object-oriented real-time computing,' Proceedings of the IEEE 1st International Symposium on Object-Oriented Real-Time Distributed Computing, Kyoto, Japan (April 1998), pp. 342-349.

[21] Object Management Group, "Fault Tolerance for CORBA,' OMG Technical Committee Document orbos/98-10-08 (October 1998).

[22] Object Management Group, "The Common Object Request Broker: Architecture and Specification,' 2.3 edition, OMG Technical Committee Document formal/98-12-01 (June 1999).

[23] Object Management Group, "Fault Tolerant CORBA,', OMG Technical Committee Document orbos/99-10-05 (October 1999).
[24] J. Schonwalder, S. Garg, Y. Huang, A. P. A. van Moorsel and S. Yajnik, "A management interface for distributed fault tolerance CORBA services," Proceedings of the IEEE 3rd International Workshop on Systems Management, Newport, RI (April 1998), pp. 98-107.

[25] E. Shokri, H. Hecht, P. Crane, J. Dussault and K. H. Kim, "An approach for adaptive fault tolerance in object-oriented open distributed systems,' Proceedings of the IEEE 3rd International Workshop on Object-Oriented Real-Time Dependable Systems, Newport Beach, CA (February 1997), pp. 298-305.

[26] R. van Renesse, K. Birman, M. Hayden, A. Vaysburd and D. Karr, 'Building adaptive systems using Ensemble,' Software - Practice and Experience, vol. 28, no. 9 (July 1998), pp. 963-979.

[27] A. Vaysburd and K. Birman, "The Maestro approach to building reliable interoperable distributed applications with multiple execution styles,' Theory and Practice of Object Systems, vol. 4, no. 2 (1998), pp. 73-80.

[28] J. Wensley, P. M. Melliar-Smith, et al, "SIFT: Design and analysis of a fault-tolerant computer for aircraft control,' Proceedings of the IEEE, vol. 66, no. 10 (October 1978), pp. 1240-1255. 